\title{
David L. McMahan, The Making of Buddhist Modernism
}

Oxford, Oxford University Press, 2008, 320 p.

\section{Marion Dapsance}

\section{(Q) OpenEdition}

\section{Journals}

Édition électronique

URL : http://journals.openedition.org/assr/26499

DOI : $10.4000 /$ assr.26499

ISSN : $1777-5825$

Éditeur

Éditions de l'EHESS

Édition imprimée

Date de publication : 31 décembre 2014

Pagination : 247

ISBN : 978-2-7132-2467-6

ISSN : 0335-5985

Référence électronique

Marion Dapsance, «David L. McMahan, The Making of Buddhist Modernism », Archives de sciences sociales des religions [En ligne], 168 | 2014, mis en ligne le 11 mai 2015, consulté le 22 septembre 2020. URL : http://journals.openedition.org/assr/26499; DOI : https://doi.org/10.4000/assr.26499

Ce document a été généré automatiquement le 22 septembre 2020.

(c) Archives de sciences sociales des religions 


\section{David L. McMahan, The Making of Buddhist Modernism}

Oxford, Oxford University Press, 2008, 320 p.

Marion Dapsance

\section{RÉFÉRENCE}

David L. McMahan, The Making of Buddhist Modernism, Oxford, Oxford University Press, 2008, $320 \mathrm{p}$. 
1 Ce que la plupart des Américains et des Européens entendent aujourd'hui par «bouddhisme » est en fait une tradition hybride datant d'environ 150 ans, qui doit tout autant aux Lumières européennes qu'à l'éveil du Bouddha, à la colonisation de l'Asie qu'à la méditation, au Romantisme qu'au canon pali. Telle est la thèse de The Making of Buddhist Modernism de David McMahan, professeur américain d'études religieuses, qui retrace les généalogies philosophiques et idéologiques ayant donné naissance, au cours des deux siècles derniers, à ce qu'il appelle « le modernisme bouddhique ». Ce terme, emprunté au bouddhologue Heinz Bechert, désigne un développement récent et distinct dans l'histoire du bouddhisme, qui met l'accent sur la compatibilité avec la science, la

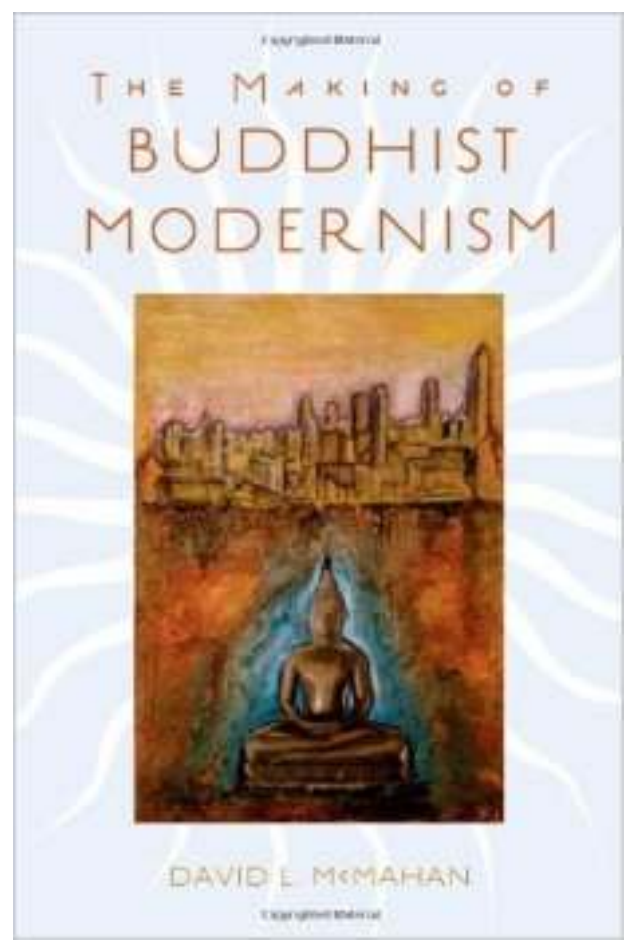
rationalité, l'autonomie et la pratique de la méditation au détriment des textes canoniques, des institutions traditionnelles et des pratiques rituelles et dévotionnelles. Cette modernisation, par acclimatation sélective aux théories dominantes et aux valeurs implicites des Occidentaux, est présentée par ses porte-paroles comme un "retour au bouddhisme originel », alors qu'elle s'écarte résolument des conceptions et des pratiques bouddhiques asiatiques. Donald Lopez avait déjà décrit ces tendances dans l'introduction de son Modern Buddhist Bible, publié en 2002. Le tibétologue y indique que les partisans du "bouddhisme moderne", membres des classes moyennes éduquées occidentales et asiatiques, considèrent le message original du Bouddha comme fondamentalement compatible avec « la raison, l'empirisme, la science, l'universalisme, la tolérance, la liberté et le rejet de l'orthodoxie religieuse » (p.X). Il suggère que ce bouddhisme est devenu " une école bouddhique transnationale à part entière, qui dépasse les frontières culturelles et nationales, créant [...] un réseau cosmopolite d'intellectuels écrivant la plupart du temps en anglais » (p. XXXIX, ma traduction). Cette école ne s'enracine plus dans un contexte géographique particulier, ni dans une tradition doctrinale ou rituelle ancienne : il s'agit plutôt de l'excroissance moderne de diverses écoles traditionnelles, développée à différents endroits du globe. Cette école est dotée de sa propre lignée et de ses propres textes canoniques - essentiellement les ouvrages d'auteurs populaires ou semi-académiques tels que Soen Shaku, Dwight Goddard, D. T. Suzuki, Alexandra David-Néel et, plus récemment, Shunryu Suzuki, Sangharakshita, Alan Watts, Thich Nhat Hanh, Chögyam Trungpa, Sogyal Rinpoché et le $14^{\mathrm{e}}$ Dalaï-Lama.

2 L'ouvrage de McMahan approfondit l'étude de ces tendances et tente de donner une cartographie générale de ce mouvement de modernisation transnational. Selon lui, les différents auteurs qui ont traité du phénomène prennent pour acquis, sans les décrire, les processus de modernisation, de démythologisation, de rationalisation, de psychologisation et de protestantisation du bouddhisme. Or, considère-t-il, « on a peu 
éclairci les forces idéologiques modernes spécifiques, les sources textuelles, les pratiques sociales et culturelles, les philosophies officielles et les présupposés implicites impliqués dans ces processus permanents»(p. 8, ma traduction). C'est pourquoi cet ouvrage se donne pour objectif de «mettre au jour quelques-uns des textes littéraires, des concepts, des idéologies et des pratiques modernes occidentaux spécifiques qui se sont mêlés au bouddhisme pour façonner une forme moderniste unique de dharma ", de montrer " comment les conditions de la modernité ont créé les paramètres implicites à certaines interprétations du bouddhisme plutôt qu'à d'autres " (p. 8). L'intérêt de ce livre-mais aussi sa faiblesse - est qu'il théorise le champ du modernisme bouddhique à partir d'une histoire générale des idées, sans trop laisser de place à des exemples concrets de pratiques qui, souvent, viennent contredire ou nuancer les discours avancés par les réformateurs. L'auteur est cependant conscient du risque de réduire la diversité et la complexité du phénomène et agrémente son récit de descriptions de cas particuliers d'auteurs occidentaux et de réformateurs asiatiques, qui permettent d'avoir un aperçu de la manière dont ces grandes tendances idéologiques ont été reprises et aménagées par les modernisateurs du bouddhisme, en fonction de leurs stratégies et de leurs intérêts propres (décolonisation, résistance à l'impérialisme chrétien, élaboration d'une spiritualité alternative permettant de faire face aux défis posés par la science, etc.).

3 L'ouvrage se compose de quatre parties. Il débute par une série de chapitres introductifs qui présentent l'étendue et les contours du modernisme bouddhique, ses dynamiques, ses figures majeures, que l'auteur resitue dans leur contexte historicoculturel. McMahan passe ensuite au commentaire de thématiques clés, comme le discours sur la science, le romantisme et l'appropriation de la notion d'interdépendance. Puis, il traite de la composante désormais centrale du bouddhisme modernisé, la méditation. Enfin, l'auteur s'emploie à imaginer ce que pourraient être les développements futurs de cette modernisation du bouddhisme, en évoquant ce qu'il appelle « le bouddhisme post-moderne ».

Dans ses chapitres introductifs ( 1 à 3), McMahan trace les limites, l'étendue et la portée du modernisme bouddhique. Il définit celui-ci non comme «l'ensemble du bouddhisme existant à l'ère moderne mais, plutôt, les formes de bouddhisme qui ont émergé de leur implication avec les forces culturelles et intellectuelles dominantes de la modernité " (p. 6, ma traduction). McMahan définit ces forces en recourant aux travaux de Charles Taylor, en particulier son Sources of the Self (1989), qui identifie trois grandes ressources philosophiques, religieuses et sociales de l'identité moderne: le monothéisme occidental, le rationalisme et le naturalisme scientifique, et l'expressivité romantique. En se confrontant à ces territoires idéologiques occidentaux, en projetant leur lumière sur certaines nuances particulières du bouddhisme qui pouvaient présenter quelque ressemblance, les modernisateurs, Occidentaux et Asiatiques ont inventé des réponses bouddhiques aux problèmes et aux questions dominantes de la modernité occidentale. Ils ont ainsi créé un mouvement relativement homogène, qui se concentre essentiellement sur la méditation, l'engagement social, l'internationalisation et promeut l'égalité, l'universalité, l'autonomie, tout en réduisant fortement la part du rituel, de la mythologie et de l'institution. L'auteur identifie plusieurs exemples de traditions bouddhiques nouvelles, aussi bien asiatiques qu'occidentales, mais sa focale reste essentiellement le bouddhisme nord-américain. Il justifie ce choix par le fait que l'anglais est devenu la « lingua franca du bouddhisme moderne» (p. 21), le fait que de nombreux enseignants bouddhistes vivent aujourd'hui aux États-Unis et enfin que ce 
pays serve de «laboratoire pour les adaptations, les innovations et les reformulations du bouddhisme en termes modernistes » (p. 22).

Dans le chapitre 2, l'auteur s'emploie à dresser une typologie des différents modes d'affiliation au bouddhisme moderne grâce à une série de portraits variés de pratiquants: la sympathisante occidentale, la pratiquante laïque thaïlandaise, l'enseignante américaine, le moine tibétain traditionnel, le modernisateur asiatique. La succession de ces portraits montre que « la ligne de démarcation entre un moderniste et un traditionaliste est souvent floue et irrégulière ». S'il existe en effet « de profondes différences entre les formes extrêmes des bouddhismes traditionnel et moderniste ", ces deux courants sont quelquefois intimement liés (p. 27-28). Par exemple, les deux pratiquantes laïques, occidentale et thaïlandaise, ont en commun de « combiner à leur pratique bouddhique des éléments non bouddhiques venus de leurs cultures respectives, dont elles ne sont la plupart du temps pas conscientes» - la psychothérapie pour l'occidentale, le culte des esprits pour la thaïlandaise (p. 37). Pourtant, leur approche du bouddhisme semble ne rien avoir en commun, l'une privilégiant la lecture d'ouvrages théologiques et la méditation solitaire, l'autre la pratique rituelle au bénéfice de sa famille. De même, il n'est pas rare de voir des sympathisants occidentaux adhérer à la croyance aux êtres surnaturels, un trait que l'on pourrait a priori rattacher aux modalités traditionnelles de la pratique religieuse. Le cas du modernisateur asiatique montre, en outre, qu'il n'est pas possible d'assimiler bouddhisme moderne et bouddhisme occidental.

6 En l'absence d'équivalence entre ces deux dernières catégories, McMahan identifie trois caractéristiques permettant de distinguer le bouddhisme moderne du bouddhisme traditionnel : la dé-traditionalisation (le tournant subjectif qui détourne des autorités transcendantes extérieures), la dé-mythologisation (terme emprunté à Heinz Bechert) et la psychologisation. McMahan montre que l'adaptation à la modernité est réalisée à travers un processus complexe de "décontextualisation et de recontextualisation", dans lequel les traditions bouddhiques sont passées au filtre de nouveaux « réseaux de sens, de valeurs et de pouvoirs» (p.62). Dans les chapitres 4 à 6, l'auteur montre l'émergence d'un dialogue inédit entre certaines notions bouddhiques traditionnelles et les trois grandes ressources de l'identité moderne définies par Taylor. Il explore plus particulièrement le cas des discours scientifique et romantique, qui ont influencé, entre autres, les conceptions de l'éveil. McMahan souligne que, dans les deux cas, le bouddhisme réformé a à la fois adopté et remis en cause les caractéristiques de la modernité. Il a ainsi repris à son compte les grandes lignes du rationalisme scientifique, tout en rejetant la vision du monde matérialiste et utilitariste qui lui est souvent associée. Cette tension entre sympathie et répugnance à l'égard des thématiques modernes occidentales est, selon l'auteur, une caractéristique inhérente au processus de modernisation du bouddhisme - comme à la constitution de toute forme de "modernité indigène ». Elle permet d'expliquer l'émergence de courants divers au sein de ce mouvement global du modernisme bouddhique.

7 Le chapitre 4 s'intéresse au discours du bouddhisme scientifique, aujourd'hui central dans la définition moderne de ce dernier. McMahan identifie «deux préoccupations différentes mais qui se chevauchent, suscitées par deux crises de légitimité dans des contextes culturels disparates » (p. 90) : le colonialisme et « la crise de foi victorienne ». Le premier est analysé à travers la figure d'Anagarika Dharmapala, la seconde à travers les trajectoires d'Henry Scott Olcott et de Paul Carus. Le discours sur la science a été 
utilisé, dans le premier cas, comme une arme politique et culturelle destinée à lutter contre l'impérialisme chrétien au Sri Lanka, dans le second, comme réponse au défi posé par le darwinisme et la pluralité religieuse. Ces objectifs distincts se sont rejoints avec la collaboration d'olcott et de Dharmapala, jusqu'à aboutir à la création conjointe d'un Catéchisme bouddhique.

Le chapitre 5 explore les liens entre le Romantisme et le modernisme bouddhique. McMahan s'intéresse ainsi au rôle de l'art, de la créativité, de la notion de nature et de la spontanéité dans l'élaboration du "bouddhisme" tel que nous le connaissons aujourd'hui. Il s'attarde sur le cas du Zen américain, popularisé par D. T. Suzuki, et sur le mouvement Beat, mais laisse de côté le rôle majeur joué par la Société Théosophique dans la reformulation du bouddhisme à l'attention des Occidentaux et du mouvement New Age de manière plus générale. Cette préférence trahit sans doute la volonté de l'auteur de s'attacher surtout à l'étude du bouddhisme américain, au détriment de l'européen.

9 Dans le chapitre 6, l'auteur s'intéresse plus particulièrement à la notion d'« interdépendance », expliquant comment les courants romantiques et rationalistes ont contribué à en développer une approche moderniste, radicalement différente du concept traditionnel de «coproduction conditionnée » (sanscrit pratîtyasamutpâda). En effet, les partisans $\mathrm{du}$ bouddhisme moderne considèrent aujourd'hui l'« interdépendance » sous un angle social, économique et écologique global inédit, la définissant comme la nécessité, pour tous les êtres humains, d'agir de concert, dans le respect, l'harmonie et la paix. Or, le concept traditionnel de «coproduction conditionnée » n'a pas pour objet la valorisation de la collaboration humaine ni même une conception holiste de l'univers. Il est plutôt un outil épistémologique permettant au pratiquant de réaliser la vacuité des phénomènes et de se défaire de son attachement au monde extérieur. Ainsi, le modernisme bouddhique a impliqué une inversion radicale de la valeur accordée au monde : d'objet à fuir, ce dernier est devenu un habitat commun à préserver et à améliorer. De là les nouvelles préoccupations sociales et écologiques de certains courants qui se prévalent du bouddhisme moderne.

Les chapitres 7 et 8 sont consacrés au mouvement de la méditation. Dans le septième chapitre, McMahan décrit le contexte culturel et philosophique qui a donné naissance $\mathrm{au}$ « tournant subjectif » et revient sur certaines notions modernes fondamentales qui ont donné naissance aux traits aujourd'hui majeurs du bouddhisme contemporain. La «méditation » est ainsi décrite comme le produit commun de tendances occidentales particulières (à la fois la référence romantique à l'intériorité comme source de vérité et l'examen rationnel des phénomènes naturels promu par le rationalisme) et de certaines pratiques psychocorporelles dissociées de leur contexte rituel, social et cosmologique traditionnel par certains réformateurs asiatiques. Cette dissociation est l'une des grandes caractéristiques du bouddhisme moderne. Il est intéressant de souligner le caractère inattendu des liens qu'entretiennent aujourd'hui bouddhisme et méditation : tout en étant presque entièrement privée de références proprement bouddhiques, jusqu'à pouvoir être pratiquée par des chrétiens, des Juifs ou des athées, la méditation est paradoxalement considérée comme la pratique centrale, nécessaire et suffisante, du bouddhisme (p.185). Il est cependant regrettable que l'auteur ne définisse pas ce qu'il entend concrètement par "méditation », tant il est vrai que cette expression recouvre aujourd'hui des pratiques diverses. 
11 Dans le chapitre 8, McMahan poursuit sa discussion sur la méditation en s'intéressant plus particulièrement à l'une de ses facettes les plus récentes, appelée "pleine conscience " (mindfulness). Les pratiques qui se rattachent à cette dénomination dérivent, selon l'auteur, d'une mentalité purement occidentale. En revenant sur la notion romantique d'épiphanie (p.121-122), il établit des parallèles entre les pratiques d'observation de la vie quotidienne et de la conscience décelables dans la littérature du début du $\mathrm{xx}^{\mathrm{e}}$ siècle (chez Virginia Woolf, James Joyce, Hermann Hesse et Marcel Proust). Ces auteurs auraient contribué à sensibiliser le public aux questions de la sensibilité et $\mathrm{du}$ for intérieur, jusqu'à donner naissance à des pratiques sociales en dehors de la seule sphère littéraire. Le roman Siddhartha aurait par ailleurs joué un rôle crucial dans la formation et la diffusion des représentations occidentales désormais admises du bouddhisme. Parmi les auteurs occidentaux à succès, McMahan aurait aussi pu mentionner Alexandra David-Néel, qui contribua sans doute bien plus que Hesse à la popularisation du bouddhisme moderne.

Dans son dernier chapitre, McMahan s'intéresse à l'avenir du modernisme bouddhique, qu'il dénomme, sans trop de précisions, « bouddhisme postmoderne ». Outre le fait qu'il s'aventure sur les sentiers dangereux de la prospective, ce chapitre présente le défaut de dissoudre la dimension globale du phénomène - alors que l'auteur refusait jusque-là d'assimiler bouddhisme occidental et bouddhisme moderne - pour ne plus s'intéresser qu'à ses formes occidentales. Les questions de la démocratisation ou de la féminisation $\mathrm{du}$ bouddhisme, que McMahan présente comme des évolutions évidentes du bouddhisme modernisé d'Occident, restent cependant posées pour ce qui est du bouddhisme asiatique, où ce type de transformations semble beaucoup moins assuré. En outre, en Occident même, les approches ethnographiques signalent que, dans certains groupes, notamment d'obédience tibétaine, les discours sur la modernisation, la psychologisation ou la féminisation ne sont pas suivis d'effets - à moins qu'il ne s'agisse d'effets inverses. On assiste en effet souvent à une résurgence des instances de pouvoir traditionnelles en lieu et place de la « modernisation » revendiquée. Bien qu'il évoque le caractère persistant du traditionalisme tibétain en Occident (ou «retraditionalisation »), McMahan ne parvient pas à s'extraire des discours apologétiques tenus par les acteurs qu'il étudie. Il aurait fallu, pour ce faire, s'intéresser aux pratiques effectives au moins autant qu'aux doctrines, aux idées et à la propagande. C'est en effet le reproche que l'on pourrait faire à cet ouvrage, qui par ailleurs offre une excellente grille de lecture du bouddhisme moderne en termes d'histoire des idées : les courants intellectuels occidentaux et asiatiques qui ont participé à l'élaboration du modernisme bouddhique sont bien identifiés, leurs liens logiques élucidés, mais l'on voit mal, concrètement, socialement, comment ils ont donné lieu à des définitions et à des pratiques hybrides nouvelles. 